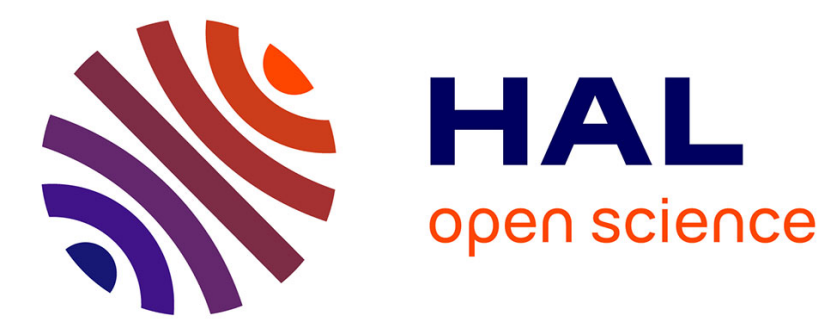

\title{
L'aventurier, une figure de la migration africaine Sylvie Bredeloup
}

\section{To cite this version:}

Sylvie Bredeloup. L'aventurier, une figure de la migration africaine. Cahiers Internationaux de Sociologie, 2008, Vol. CXXV (n 125), p. 281-306. ird-02878798

\section{HAL Id: ird-02878798 \\ https://hal.ird.fr/ird-02878798}

Submitted on 23 Jun 2020

HAL is a multi-disciplinary open access archive for the deposit and dissemination of scientific research documents, whether they are published or not. The documents may come from teaching and research institutions in France or abroad, or from public or private research centers.
L'archive ouverte pluridisciplinaire HAL, est destinée au dépôt et à la diffusion de documents scientifiques de niveau recherche, publiés ou non, émanant des établissements d'enseignement et de recherche français ou étrangers, des laboratoires publics ou privés. 


\title{
L'aventurier, une figure de la migration africaine
}

\author{
Par Sylvie Bredeloup, directrice de recherches à l'IRD (UMR LPED Marseille) \\ Sylvie.Bredeloup@up.univ-mrs.fr
}

Ces dernières années, les ressortissants de l'Afrique subsaharienne, qui traversent le désert et les détroits, occupent régulièrement la scène politique euro-méditerranéenne. Ces migrants sont de plus en plus souvent qualifiés d'aventuriers par les médias quand leurs trajectoires ne sont pas hâtivement assimilées à celles de «desperados », ces hors-la-loi animés de passions violentes. Mais que peut-on dire de ces assignations ? Ont-elles un contenu objectif ou relèvent-elles simplement d'un exercice visant à criminaliser le migrant ? En outre, font-elles écho à des désignations emic et, dans cette perspective, à quels processus d'identification correspondent-elles ? Autrement dit, on peut se demander à quoi ressemble l'aventure migratoire et si l'aventurier constitue une figure nouvelle dans l'histoire de la migration africaine depuis que des turbulences répétées affectent en profondeur sur le continent autant les conduites individuelles, les codes moraux et les imaginaires sociaux que les structures sociales, économiques et politiques de la société. Ou bien, l'aventurier se pose en une figure récurrente, qui qualifie depuis longtemps déjà certaines formes migratoires, mais qui connaîtrait un regain de visibilité et peut-être d'activité, à mesure que les politiques migratoires se durcissent un peu partout sur la planète et que la libre circulation des hommes est rendue de plus en plus problématique. Enfin, entendue au sens de carrière, c'est-à-dire d'une succession de passages d'une position à une autre accomplie au sein d'un système, si l'aventure a un commencement, elle a aussi une fin et, dans cette perspective, seront considérées les différentes modalités de sorties de l'aventure.

\section{Aventure, aventurier : quelles représentations sur la scène occidentale ?}

Sociologues, philosophes et historiens semblent s'être moins intéressés à l'aventure en tant qu'événement qu'aux processus de désignation par lesquels un événement peut être ou non considéré comme aventure. Selon G. Simmel, qui a été le premier à prendre pour objet d'étude l'aventure, c'est l'intensité avec laquelle est vécue un événement qui peut ou non le transformer en aventure et mieux nous faire ressentir le piment de la vie. "La réalisation d'un contenu propre à une aventure ne constitue pas encore une aventure. Le fait d'avoir encouru un danger de mort ou d'avoir conquis une femme pour un bonheur éphémère, le fait que des facteurs inconnus, qui ont été mis en jeu, ont apporté un gain surprenant ou une perte inattendue... tout cela ne constitue pas encore nécessairement une aventure, et ne le devient que par une certaine tension du sentiment vital dans la réalisation de pareils contenus $» . .$. "[L'aventure] est avant tout caractérisée par le radicalisme avec lequel elle se manifeste comme étant une tension caractéristique de la vie. Par l'intensité de ces tensions, l'événement ordinaire devient une aventure ${ }^{1}$. Contrairement donc à certaines définitions qu'on peut retrouver dans des dictionnaires, l'aventure ne serait pas nécessairement un événement inattendu, extraordinaire, mêlant le danger au plaisir de la découverte.

En outre, selon Sartre notamment dans «La Nausée », c'est la mise en récit qui permettrait d'élever un événement même ordinaire au rang d'aventure. «... J'ai cru qu'on pouvait définir l'aventure: un événement qui sort de l'ordinaire, sans être forcément

\footnotetext{
${ }^{1}$ La métaphore de l'aventure, p. 82 et 87.
} 
extraordinaire. On parle de la magie des aventures... Il m'est arrivé des histoires, des événements, des incidents, tout ce qu'on voudra. Mais pas des aventures. Ce n'est pas une question de mots ; je commence à comprendre... Les aventures sont dans les livres [...] : pour que l'événement le plus banal devienne une aventure, il faut et il suffit qu'on se mette à le raconter. C'est ce qui dupe les gens : un homme, c'est toujours un conteur d'histoires, il vit entouré de ses histoires et des histoires d'autrui, il voit tout ce qui lui arrive à travers elles; et il cherche à vivre sa vie comme s'il la racontait. ». P. Mac Orlan a souligné, de son côté, le «paradoxe de l'aventurier»: il n'existe pas en tant que tel mais ne serait que recréation $a$ posteriori par la volonté d'une société bourgeoise, avide de rêves et d'exploits. Des rapprochements peuvent également être établis avec la manière dont H.S. Becker a défini la déviance comme le produit d'un processus intégrant la réponse des autres individus aux conduites déviantes. Dit autrement, le caractère aventureux d'un acte dépendrait autant de ce que les uns et les autres en disent et de la manière dont ils le disent que de la nature même de l'acte. Ce n'est pas parce que quelqu'un se pense aventurier que les autres vont nécessairement le percevoir comme tel et, réciproquement, ce n'est pas parce que quelqu'un ne se considère pas comme aventurier qu'il ne peut pas être désigné comme tel par autrui.

Pour nombre d'auteurs, au-delà de ce processus d'objectivation incontournable, l'aventure se définit également en relation, en comparaison avec d'autres états. Pour G. Simmel, c'est la présence simultanée d'impressions contraires dans la vie psychique qui caractérise l'aventure : "La certitude que nous avons du résultat d'une action, donne à cette action plus une coloration qu'une qualité particulière. Si, au contraire, nous ne sommes pas sûrs d'arriver à notre but, si nous sommes conscients de l'incertitude du succès, non seulement notre sentiment de sécurité diminue par là en intensité, mais notre attitude dans la pratique se trouve être déterminée intérieurement et extérieurement d'une façon toute spéciale. L'aventurier se comporte vis-à-vis de ce qui est irrationnel dans la vie, comme nous nous comportons d'ordinaire vis-à-vis de ce qui est calculable à l'avance ». Un demi-siècle plus tard, on retrouve une construction voisine mais transposée dans le temps chez $\mathrm{V}$. Jankelevitch qui définit le "pathos de l'aventure comme un complexe de contradictions ». Comparée à l'ennui et au sérieux, l'aventure est entendue comme une autre manière de considérer le temps. "Ce qui est vécu, et passionnément espéré dans l'aventure, c'est le surgissement de l'avenir. L'ennui, par contre, est vécu plutôt au présent [...] si l'aventure se place surtout au point de vue de l'instant, l'ennui et le sérieux considèrent le devenir surtout comme intervalle : c'est le commencement qui est aventureux, mais c'est la continuation qui est, selon les cas, sérieuse ou ennuyeuse. Il s'ensuit naturellement que l'aventure n'est jamais "sérieuse » et qu'elle est a fortiori recherchée comme un antidote de l'ennui »².

Quant aux historiens, qui ont travaillé sur les discours sur l'aventure à travers les romans, ils montrent également comment les définitions ont évolué dans le temps, renseignant sur les sociétés qui les mettent en œuvre et sur les utopies qui les traversent. Présenté tour à tour dans l'histoire occidentale comme un mercenaire des armées de la fin du moyen-âge, (Roch, 2002), puis comme un corsaire des mers de l'Amérique, un flibustier au XVIIème siècle (Ouellet, 1996), un intrigant, un usurpateur à l'image de Casanova ou de Cagliostro au milieu du XVIIIème siècle, ou enfin comme un chercheur d'or, un trafiquant ou un explorateur au milieu du XIXème siècle, l'aventurier demeure un personnage négatif. Au XIVème siècle, la première définition enregistrée renvoie à celle d'un étranger dangereux, à la mauvaise réputation dont on ne comprend pas la langue et dont on craint les débordements. Les dictionnaires le présentent par la suite comme un individu solitaire, déterminé, peu

\footnotetext{
${ }^{2}$ L'aventure, l'ennui et le sérieux, p.7 (version de 1963).
} 
scrupuleux, explorant les espaces lointains et mystérieux, vivant d'expédients, ayant le goût du risque, affrontant les dangers et cherchant à contourner les règles édictées par la société. Il faut donc attendre le milieu du XIXème siècle pour que, dans la littérature occidentale, l'image de l'aventurier soit pleinement revalorisée (Venayre, 2002). L'homme devient « doué pour le vagabondage »; l'accomplissement de soi, la saisie de son propre destin, l'exploration du sens caché du monde sont alors érigés en vertus. Les discours exaltent l'aventure pour l'aventure à une époque où la figure du bourgeois commence à être stigmatisée et où les représentations de l'espace de la planète connaissent une mutation radicale. Les terres vierges, lointaines et inconnues se raréfiant du fait de la modernisation des transports, les figures de l'aventure s'apparentent désormais à celles de la nostalgie (Venayre, 2002). Au XXème siècle, l'engouement pour les pratiques d'escapade s'intensifie encore à mesure que les grandes idéologies reculent, que les déclassements sociaux se multiplient et que l'individualisme, le désir d'autonomie et la relance d'un certain hédonisme s'exacerbent. À l'heure où le défi s'est transformé en vertu cardinale, l'aventure, qui rime désormais avec libéralisme économique et, qui est étroitement associée à l'entreprise, peut être vécue au coin de la rue ${ }^{3}$, sur le temps du loisir, à travers une mise en jeu risquée du corps, sur un mode acrobatique ou sportif, permettant d'échapper momentanément, par l'action ou le rêve, au quotidien maussade (Pociello, 1987). Devenue une véritable institution, la néo-aventure se lie dorénavant au monde du commerce et de l'industrie ; l'aventurier se pose en professionnel du spectacle dont la recherche de l'extrême, les exploits calculés et médiatisés sont nécessairement époustouflants pour jouer pleinement leur rôle économique attendu auprès des sponsors ou mécènes (Le Breton, 1996 ; Ehrenberg, 1998).

Reste à savoir si les figures emblématiques de l'aventurier occidental comme les discours tenus en Europe et aux États-Unis à propos de l'aventure font sens chez leurs cousins africains ${ }^{4}$ qui n'ont pas été inondés de littérature de voyages et d'aventures. On peut en effet douter que l'aventurier africain soit l'héritier de séquences historiques reliant le chevalier médiéval au flibustier et à l'intrigant de l'Ancien régime. On peut s'interroger également sur les liens imaginaires qu'il peut entretenir avec le conquistador à la conquête du nouveau monde, avec le cowboy du Far West, avec le Hobo, ce nomade ouvrier parcourant les routes à la recherche d'un travail pendant la Grande Dépression (Anderson, 1993), avec le vagabond de la Beat Generation ou encore avec le survivant de la guerre du Vietnam ou enfin le sportif de l'extrême. Assiste-t-on, ces dernières décennies en Afrique subsaharienne, à une valorisation culturelle du défi et du risque notamment à travers la migration interne comme internationale ? Célèbre-t-on de nouveaux modèles de réussite construits autour de l'audace et de l'innovation? Et dans cette perspective, sur quels imaginaires, sur quelles mythologies s'appuie-t-on pour légitimer leur diffusion?

\section{En Afrique, quand le migrant aventurier prend le pas sur le travailleur immigré}

\section{Travailleur saisonnier, travailleur immigré : les deux facettes éclairées de la migration africaine}

Historiquement, la migration africaine a été appréciée essentiellement à travers l'étude des flux ; l'analyse des causes puis des conséquences de ces mouvements a été privilégiée au détriment d'une réflexion sur les profils et carrières de migrants. Et quand ces dernières ont

\footnotetext{
${ }^{3}$ Allusion à l'ouvrage de P. Brückner et de A. Finkielkraut, « au coin de la rue, l'aventure ».

${ }^{4}$ Il faudrait également dissocier les figures littéraires de l'aventurier qui se sont construites en Europe des grands récits élaborés dans les Amériques et au Canada bien que le point de vue européen-centré ait pendant longtemps orienté l'histoire occidentale et occulté certaines trajectoires (Andrès, 2004).
} 
été étudiées, les auteurs se sont le plus souvent limités à éclairer celles du travailleur saisonnier qui se rend ou non à la demande d'une institution dans un pays frontalier pour mettre en valeur sa terre ou son sous-sol et celle du travailleur immigré, parti rejoindre en Europe les chantiers industriels à l'appel d'une ancienne puissance coloniale. Dès le milieu du XIXème siècle, des migrants saisonniers rebaptisés «navétanes » ${ }^{5}$, originaires du Soudan, de Guinée française et de Guinée portugaise rejoignaient, à chaque hivernage nouveau, en Sénégambie, d'autres ressortissants du Sénégal et de Gambie et de Mauritanie pour cultiver l'arachide. Ce phénomène social d'ampleur largement décrit par $\mathrm{P}$. David a concerné des millions d'Africains pendant le troisième quart du XIXème siècle. L'usage du terme « immigré » est devenu tellement familier qu'il s'est imposé presque «naturellement », sans qu'il soit nécessaire d'expliciter l'implicite d'une telle notion (Spire, 1999). La figure sociale de l'immigré a donc été systématiquement associée au travail, et plus particulièrement au travail non qualifié. L'Africain en France fut assimilé pendant plusieurs décennies à l'ouvrier spécialisé, sahélien illettré qui travaillait dans les usines automobiles de la région parisienne et qui résidait seul dans les foyers type Sonacotra. Mais en lien avec la crise économique, l'assignation de l'immigré à sa seule fonction de travailleur est devenue moins prégnante ; l'immigré est devenu celui qui a quitté son pays pour s'installer durablement sur le territoire de l'autre.

Contrairement aux idées reçues, la migration n'est pas seulement déterminée par la misère et le danger. Autrement dit, les déséquilibres économiques entre les continents, la pression démographique dans certaines régions d'Afrique, les turbulences politico-militaires en Afrique ne justifient pas à eux-seuls les départs de population. Les motivations individuelles sont aussi essentielles; l'ambition personnelle est un moteur important qui conduit sur les routes les éléments les plus intrépides.

\section{Du marin colonial aux ghettomen en passant par les verandah boys et les sapeurs... les acteurs de l'aventure migratoire africaine}

En dépit de la multiplicité des formes prises par l'aventure au cours des siècles, le marin est l'une des premières figures historiques de l'aventurier, l'homme venu d'ailleurs. À l'ère de la marine à voile, la littérature a largement contribué à mettre en regard désir d'aventure et métiers de la mer. Pour «Lord Jim » de J. Conrad notamment, seule la vocation de marin est promesses d'aventures. R. Mathé parle d' " aventure quêteuse » pour qualifier à la fois les parcours du voyageur, du marin, de l'explorateur et du pionnier, considérant avant tout l'aventurier comme un curieux au sens où l'entendait Pascal (« ceux qui appartiennent à l'ordre de l'esprit ») qui souhaite satisfaire coûte que coûte son désir d'ailleurs. Le marin africain, mobilisé ou non pour l'effort de guerre à l'occasion des deux conflits mondiaux, qui débarque sur les côtes françaises peut-il être considéré comme un aventurier ? Sa trajectoire relève-t-elle du mythe ou de la pratique ordinaire ? De Marseille aux villages du fleuve Sénégal, dans les années 1940-1950, les griots racontaient comment un certain Magatte Louis Ndiaye, dit Magatte Colossal, avait volé un bateau dans le port phocéen, avec la complicité d'un groupe de francs-tireurs, pour aller le livrer à la mafia du côté de Gibraltar après avoir organisé un trafic de faux papiers, destiné à permettre l'embauche dans la marine marchande à des clandestins africains débarqués en France entre les deux guerres (Bertoncello, Bredeloup, 2004). À l'image de ce « pirate » saint-louisien, quelques marins coloniaux, ressortissants de l'AOF ou de l'AEF ont vécu des moments forts dans leur carrière, s'infiltrant d'abord

\footnotetext{
${ }^{5} \mathrm{du}$ wolof nawet qui signifie hivernage, la saison d'hiver.
} 
clandestinement sur les bateaux ${ }^{6}$ des compagnies Paquet ou des Messageries Maritimes à partir de Dakar puis essuyant les tirs de la Kriegsmarine en pleine guerre mondiale à bord d'un navire armé de la marine marchande. Quelques navigateurs au long cours seulement sont parvenus au fil des années à s'enrichir grâce à des trafics de marchandises et d'hommes. La majorité, analphabète et très peu qualifiée, n'a pas su trouver un second souffle après la diésélisation des navires et a vécu dans une extrême précarité. Soutiers, mécaniciens ou serveurs à bord de paquebots - ces nouveaux «autobus de l'océan » - ils peinent à confronter leurs imaginaires de l'ailleurs à l'épreuve de la réalité. Pour les derniers marins africains encore en vie, ayant élu domicile à Marseille et tributaires d'une maigre retraite, l'aventure se résume dorénavant à une promenade quotidienne ponctuée d'escales entre les cafés PMU et les jardins du centre-ville (Bredeloup, 2004).

Jean Rouch fait partie des tous premiers anthropologues à avoir mis en évidence les déterminants psychosociologiques de la migration africaine. Dans son travail, mené d'abord au Ghana puis en Côte d'Ivoire ${ }^{7}$, à la veille des Indépendances africaines, il conte l'histoire de jeunes néo-citadins à la recherche d'exploits nécessaires à l'accomplissement de soi. Les «verandah boys », qui se rebaptisent les «Jaguars", en référence aux berlines du constructeur anglais qui les font fantasmer, se reposent sous les arcades des maisons de commerce dans la ville d'Accra en attendant de prendre la route pour l'Europe. De la même manière, les dockers et manœuvres sans emploi, d'origine nigérienne, installés provisoirement dans le quartier de Treichville d'Abidjan (Côte d'Ivoire), rêvent à un avenir meilleur et plus prestigieux. Dans cette perspective, chaque dimanche, ils regardent au cinéma les westerns mettant en scène Buffalo Bill ou les «justiciers du Far West », ainsi que les retransmissions des combats de Ray Sugar Robinson, l'un des plus grands boxeurs noirs américains de tous les temps. Dans les années 1957-1959, un peu plus au Sud de l'Afrique, du côté de Léopoldville, se développe le mouvement billiste (Kolonga, 1979). Des petits gangs se créent à l'initiative des jeunes issus des quartiers populaires. La ville africaine devient le lieu fantasmé de réalisation de leurs aspirations. Puisant également leur inspiration dans le registre des westerns américains pour se construire des noms de guerre, ils reconfigurent ainsi leur vision du territoire, de la frontière et de la chasse. Les «écumeurs du Far West» ou les «Yankees de Ngiri-Ngiri » renomment Texas ou Dallas, les écoles et les marchés de la capitale congolaise. Combinant traditions réinventées avec des références à une modernité supposée, incarnée par le Far West américain, ils inventent aussi leurs propres rites d'initiation comme ils déploient un style de vie critique à l'égard de la colonisation belge (De Boeck, 2001). On retrouve d'ailleurs sensiblement les mêmes ingrédients dans le film intitulé «Retour d'un aventurier », réalisé en 1966 par le nigérien Moustapha Alassane et qui peut être considéré comme l'un des rares westerns africains. Après un séjour aux Etats-Unis, le héros rentre au pays avec dans sa valise des panoplies de cow-boy; il constitue un gang avec des amis, lesquels se travestissent et empruntent des surnoms dignes du Far West (Black Cooper, James Kelly...). Ainsi parés, ils partent tous à l'assaut des villages environnants.

Installés, de passage ou de retour dans des cités africaines en pleine construction, ces jeunes seraient en quelque sorte les ancêtres des «Aventuriers», ces «marginaux de

\footnotetext{
${ }^{6}$ Ces récits récoltés dans la cité phocéenne ne sont pas sans rappeler certaines séquences développées dans «Bourlinguer », un ouvrage de Blaise Cendrars qui raconte comment au $17^{\text {ème }}$ siècle, à Venise, un jeune garçon débuta sa carrière de marin comme passager clandestin à bord d'un bateau à destination de Smyrne.

${ }^{7}$ Outre des articles sur les migrations saisonnières du Niger au Ghana, J. Rouch a réalisé deux films sur cette thématique. L'un intitulé «Jaguar » sorti en 1967 s'apparente à une « road movie à pieds », engagée par trois amis qui espèrent faire fortune en Gold Coast avant de regagner leur village. L'autre long métrage « Moi, un noir à Treichville » raconte les déboires et les rêves de jeunes résidant à Treichville en 1957.
} 
1'émigration africaine en France ${ }^{8}$ dont J.-D. Gandoulou s'est employé à dresser le portrait et les «arts de faire » entre «Paris et Bacongo », vingt-ans plus tard. Les premières tentatives d' «Aventure », en direction de Paris, qui datent de la fin des années 1970, s'opèrent pour les uns par étapes successives et pour les autres directement par avion. Cette émigration concerne, dans un premier temps, des garçons déscolarisés, originaires pour la majeure partie d'entre eux du quartier Bacongo à Brazzaville. Ces jeunes désœuvrés envisagent le déplacement vers la France comme un voyage initiatique, un parcours ritualisé leur permettant d'acquérir la "gamme » de vêtements de luxe qui leur assurera la consécration une fois de retour au pays. Leur émigration est essentiellement culturelle, leur mode de vie se caractérise par l'excentricité. La SAPE ${ }^{9}$ ou l'élégance éclatante, symbole de l'Occident, est envisagée comme le raccourci le plus sûr pour accéder à la réussite sociale et Paris est considéré comme le centre mondial de la Sape. Le «sapeur» est un futur aventurier dans le sens où c'est seulement à partir du moment où il a pu accomplir un retour glorieux au pays après un séjour fructueux dans la capitale française qu'on lui attribue cette appellation. "L'Aventurier est la forme achevée du sapeur» (Gandoulou, 1989). La réalisation de soi prime sur le projet économique si bien que la recherche d'un emploi stable en France ne fait pas partie des scénarii envisagés ; l'aventurier désapprouve totalement le comportement des migrants sahéliens qui «traînent un balai ou qui sont juchés sur une benne », préférant resquiller, grappiller, se débrouiller, recourir à l'économie de cueillette quand il ne parvient pas à trouver un travail ponctuel. "Vivre en aventurier est trop absorbant, exige trop de l'individu pour qu'il fasse place à un autre ordre de préoccupations ». Si les sapeurs partagent le même espace géographique que les étudiants, les fonctionnaires et les travailleurs africains, leurs pratiques sont en total décalage; ils évoluent dans un monde imaginaire. La société congolaise ne retient d'ailleurs que l'aspect exhibitionniste de leur démarche aventurière. Lors de leurs «descentes » à Brazzaville, ceux qui se rebaptisent alors les «Parisiens » circulent, paradent en groupe dans leur quartier d'origine, sapés, parfumés et grimés. Un quotidien congolais parlait de «spectacle scandaleux » pour rendre compte de la présence au stade de la Révolution de ces migrants de retour, à l'occasion d'un grand derby : «Maquillés à outrance (peau couleur jaune papaye bien mûre), les cheveux coupés à ras et noircis au cirage, noyés dans de vieux costumes achetés dans les marchés aux puces de Paris, petite cravate au cou, démarche cambrée en file indienne, ces aventuriers se sont pavanés sur les gradins de la tribune d'honneur $\gg{ }^{10}$.

Au fil des années, le mouvement de la Sape s'est réorienté progressivement vers des commerces sans patente ou des négoces délictueux (Bazenguissa, MacGaffey, 1995). Ces trafics rémunérateurs permettent aux ressortissants des deux rives du Congo de dépenser rapidement dans les nganda - ces bars à la mode zaïroise - l'argent gagné pour «vivre intensément » et acquérir ainsi prestige et réputation à l'occasion de ces actions d'éclats, à l'intérieur d'un petit milieu qu'ils ont eux-mêmes façonnés. Le risque est considéré comme un mode de vie. Ces auteurs définissent également ces commerçants qui viennent à Paris pour acquérir un nouveau statut comme des aventuriers. "The principal termes used to refer to the migration experience and the activities it involves are "l'aventure" and "la débrouillardise". "L'aventure" entails the use of physical and mental kills, and even sometimes putting one's life in danger. In "l'aventure", migrants do not stake assets which are exterior to them » (Bazenguissa, MacGaffey, 2000). Autrement dit, ceux que A. Mabanckou classe dans la catégorie des «bouillants », dans son roman «Bleu-Blanc-Rouge», cherchent par voie détournée à parvenir rapidement au faîte de leur carrière. À l'exemple de Moki alias Marcel

\footnotetext{
${ }^{8}$ Voir la préface rédigée par J. Rouch de l'ouvrage de J.-D. Gandoulou.

${ }^{9}$ Société des Ambianceurs et des Personnes Elégantes.

${ }^{10}$ Mweti du 8 septembre 1981.
} 
Bonaventure, ce sont les nouveaux acteurs de la société africaine qui ont opté pour la débrouillardise.

De la même manière que leurs confrères congolais, les ghettomen, ces jeunes citadins rencontrés par E. De Latour à Abidjan et à San Pédro, et qui s'auto-désignent par les termes d'aventuriers, de vagabonds ou de bandits, ont soif de reconnaissance et de respectabilité dans une société qui rejette les maillons les plus faibles. Ils sont à la recherche d'exploits en Côte d'Ivoire mais surtout en Europe, lesquels leur permettront de s'élever et de s'accomplir. Le détour par l'Occident devient incontournable pour ces Benguistes ${ }^{11}$ qui sont prêts à tout pour se forger une nouvelle identité plus valorisante. Influencés par le contexte néo-libéral où la recherche sauvage du profit par l'individu solitaire est érigée en valeur suprême, ces «nouveaux aventuriers de l'Europe» ont bien l'intention de "grandir leur [son] nom par n'importe quel moyen », en restant «chacun dans son chacun». Etre en zigueï ${ }^{12}$ doit permettre de «quitter la grisaille pour le triomphe ou le mouvement» (De Latour, 1999). L'ambition personnelle est un moteur puissant qui conduit sur les routes nombre de jeunes Africains et pas seulement les plus audacieux.

À l'heure où sur le continent africain les principes du salariat et de la fonction publique sont sérieusement remis en question, des itinéraires d'accumulation inédits ainsi que de nouvelles trajectoires d'ascension sociale en phase notamment avec l'essor de l'émigration, prospèrent dans les interstices de la crise économique. Un numéro de la revue Politique Africaine paru en 2001 portant sur les «figures de la réussite » montrait qu'aussi bien à Dakar, à Nouakchott ou à Yaoundé, on assiste à une montée en puissance de styles de consommation de plus en plus ostentatoires et de nouveaux principes d'affirmation de soi, fondés sur des savoirs pratiques tels la ruse et l'arnaque (Balandier,1977). La feymania camerounaise (Malaquais, 2001) comme le tcheb-tchib mauritanien (Ould Ahmed Salem, 2001) peuvent être appréhendés comme les versions locales de cette nouvelle économie morale imprégnant les imaginaires de l'État qui valorise et légitime les parcours fulgurants, les combines et les escroqueries, autrement dit tous les registres hétérodoxes de promotion individuelle quels qu'ils soient. "Pour nombre de déclassés urbains, la réussite est souvent au bout de l'aventure et de la prise de risque » (Banégas, Warnier, 2001). Aujourd'hui, qu'il soit député, marabout, commerçant, trafiquant et pas seulement migrant, le citoyen emprunte largement les traits de l'aventurier, celui dont la trajectoire serait gouvernée tant par l'astuce, la chance, l'audace que par la bravoure.

\section{L’aventure se décline aussi au féminin}

Si l'aventure concerne en grande majorité les représentants du sexe mâle, elle se décline pourtant de plus en plus au féminin. Assurément dans la société des sapeurs, la présence féminine reste mineure; les femmes préférant attendre le retour des «Parisiens ». Mais dans leurs travaux récents, R. Bazenguissa et J. MacGaffey soulignent la place croissante que jouent les femmes congolaises et zaïroises comme gestionnaires de ngandas dans la capitale française. De la même manière, dans sa recherche sur l'émigration camerounaise en France, S. Bouly de Lesdain insiste sur le rôle clé des femmes. Et dans sa

\footnotetext{
${ }^{11}$ En nushi, langue inventée dans les quartiers populaires de Côte d'Ivoire, Beng désigne l'Europe et par extension l'Occident; les Benguistes sont donc ces migrants africains qui partent pour l'Europe. Un site d'information dédié à la diaspora africaine et ivoirienne en France, en Europe et dans le monde a été créé avec pour adresse - Lebenguiste.com - et pour logo «L'Afrique en direct de Paris ».

${ }^{12}$ Zigueï signifie aventure en nushi.
} 
typologie des profils migratoires des Camerounaises résidant en France, elle introduit la catégorie des «aventurières » c'est-à-dire des femmes déscolarisées venues chercher fortune en France au côté des «étudiantes» et des «dissidentes politiques ». Ces aventurières impliquées largement en région parisienne dans des activités d'import-export ont une parenté évidente avec les jeunes ressortissantes issues de l'Afrique forestière que nous avons rencontrées sur la scène commerciale marseillaise (Bertoncello \& Bredeloup, 2004). Tout au long de la décennie 1990, elles ont ouvert des restaurants, des commerces de produits exotiques ainsi que des salons de coiffure, du côté de Noailles, quartier délaissé par le pouvoir municipal. Quelques-unes s'auto-désignent Nanas Benz, en référence à ces Togolaises millionnaires, revendeuses de pagnes qui roulent en Mercedes Benz dans les rues de Lomé. Côtoyant en outre l'élite politique, ces dernières sont devenues sur le continent africain le symbole de la réussite féminine. Á l'image de leur modèle, elles cumulent bien souvent des parcours familiaux et matrimoniaux marqués par des ruptures : décès d'un parent, mariage traditionnel refusé ou rompu par un divorce suivi d'un départ. En Afrique, ces aventurières sont parfois dénommées «amazones ${ }^{13}$; cette désignation renseignant sur les attributs guerriers et la puissance qui leur sont associés. Pour ces femmes, la migration est envisagée comme un moyen d'émancipation et contrairement aux idées reçues, cette aventure ne rime pas systématiquement avec prostitution même si ce recours ultime peut aussi être envisagé (Barden, 1993 ; Escoffier, 2006).

\section{L'aventure et ses imaginaires}

\section{Imaginaires de la prédation et du jeu}

L'aventure, qui constitue donc une forme particulière de l'expérience migratoire, doit être aussi interrogée dans ses déterminations imaginaires. L'aspiration à l'Ailleurs se décline, en effet, selon plusieurs directions. Pour nombre de ressortissants de l'Afrique équatoriale, c'est l'imaginaire de la prédation qui prime, celui porté par les «creuseurs de diamants ». Depuis bientôt deux décennies, nombre de Zaïrois, dépités et sans le sou, délaissent les écoles ou les champs (Tshibanza, 1986), parcourent des centaines kilomètres en direction du Sud pour aller « casser la pierre » du côté de la frontière angolaise. Pour ces migrants africains, les épreuves qui balisent la recherche des pierres précieuses font écho à celles de la chasse ou de la circoncision : dormir à la belle étoile, passer des semaines en brousse, affronter les dangers, la violence des concurrents, la souffrance physique sont jugées bénéfiques dans le sens où elles confortent leur identité virile. La vie au grand air, autrement dit, la vie d'aventures apparaît comme le gage d'un endurcissement dont le corps peut porter les stigmates. La fin justifie souvent les moyens de sorte que les pratiques illicites ou le banditisme font bien souvent partie de leurs parcours.

De la même manière que le gibier piégé par le chasseur permet de grandir et d'assurer la prospérité du clan, les diamants capturés par le prospecteur sont susceptibles d'apporter santé et fertilité à sa famille. Les diamants sont considérés comme des objets habités, animés, comme des entités sauvages qu'il s'agit d'apaiser avant de domestiquer (De Boeck, 2001). Les «chasseurs » de diamants, quant à eux, sont perçus comme des aventuriers à la fois capables de supporter la douleur, les privations et la violence et disposés - dans la perspective d'asseoir leur prestige - à consommer avec excès et à redistribuer de manière ostentatoire dès lors qu'ils ont revendu les gemmes trouvées. Donner c'est manifester sa supériorité disait M.

\footnotetext{
${ }^{13}$ À noter qu'au Canada, au XVIIIème siècle, certaines religieuses, qui par leurs actes, avaient fait preuve d'une forte implication dans la vie de la cité étaient aussi rebaptisées invariablement « amazones » ou « aventurières de Dieu ».
} 
Mauss. Dépenser de l'argent, c'est montrer qu'on a de la valeur et de l'importance. Si les milliers de diamineurs qui affluent dans la forêt angolaise aspirent tous à "devenir rapidement et facilement riches ", ils ne sont néanmoins qu'une minorité à pouvoir prétendre au statut de «nouveaux patrons » et à poursuivre leur voyage vers Lagos ou Johannesburg (Kivilu, 2001). Gagner beaucoup d'argent en très peu de temps et avec un faible investissement reste assurément de l'ordre du mythe. La majorité d'entre eux ne capitalisent rien sinon des expériences. Ce rêve d'un enrichissement considérable et instantané, c'est ce que L. Dietrich nomme «l'économie de casino» (Dietrich, 2002). E. Goffman dans les rites d'interaction explique que «Les casinos concrétisent pour l'individu la possibilité de se pousser à la limite de sa tolérance à la perte ou au gain, et lui permettent ainsi de s'éprouver avec précision, à ses yeux du moins ». Fatigué de jouer avec le feu et rentré définitivement de Zambie en 1994, un diamantaire sénégalais, rencontré quelques années plus tard à Dakar, usait de la même métaphore. "Le diamant, c'est quelque chose comme le casino, ce n'est pas tout le monde qui a la chance de le faire. J'aime faire business, acheter et revendre... Mais je ne souhaite pas reprendre cette activité. C'est trop un coup de poker. Maintenant j'ai une famille. L'aventure c'est fini ${ }^{14}$. L'aventure migratoire s'identifie totalement aux risques encourus et à l'intensité de la vie vécue ; elle permet à l'homme d'advenir et de s'aguerrir.

\section{Imaginaire de la contestation}

L'imaginaire congolais a également été inspiré par l'opposition politique avec pour cadre la Sape dont nous avons déjà plus haut esquissé les contours ; l'aventure est alors synonyme de contestation d'un ordre social (Bazenguissa, 1992). À travers le style vestimentaire adopté, les sapeurs entendent remettre en question, symboliquement, la politique des dirigeants congolais au pouvoir. Le développement de ce mouvement dans les années 1970 a été synchrone avec la fragilisation notable du système politique national et, a correspondu, plus radicalement, à la fin de l'État providence. Les anciens protégés se sont retrouvés d'un seul coup évincés de la sphère politique. À l'instar des autres exclus, c'est-àdire de ceux qui ont échoué à l'école à mesure que la sélection s'est durcie ou parce que leurs diplômes ne leur permettaient plus d'entrer comme leurs aînés dans la fonction publique, ils ont opté pour l'aventure, autrement dit pour un départ vers l'Europe. Les figures historiques de la réussite sociale - l'intellectuel et le fonctionnaire- ont définitivement été remplacées par celles des entrepreneurs ou autres marchands qui passent une partie de leur temps à étudier la société occidentale pour pouvoir mieux en contourner les procédures et y assurer leur propre réussite.

Exclus du champ politique dans leurs pays respectifs, les Congolais et Zaïrois s'évertuent à «s'exclure librement» de ce même champ au travers de pratiques ostentatoires, une fois arrivés à Paris ou Bruxelles. R. Bazenguissa et J. MacGaffey ont d'ailleurs montré qu'au fil du temps, les deux imaginaires - celui du creuseur et du sapeur pouvaient s'interpénétrer : «le creuseur devient un sapeur et le mouvement de la SAPE se tourne de plus en plus vers les trafics» (Bazenguissa, MacGaffey, 1995: 129). Éprouvant leur débrouillardise, les sapeurs des deux Congo ont mis en place des dispositifs commerciaux plus ou moins licites dans les capitales européennes, destinés à valoriser leur identité et à les légitimer en tant que membres d'une nouvelle élite. Parce qu'ils ne peuvent trouver place dans leur société d'origine, ils travaillent à redonner sens à leurs parcours de vie entre ici et là-bas. Et c'est bien cette mise en récit qui peut transformer leur trajectoire ordinaire en aventure héroïque.

\footnotetext{
${ }^{14}$ Entretien réalisé à Dakar le 5 février 1996.
} 


\section{L'épopée}

L'aventure migratoire appartient aussi parfois à une geste épique, portée par des imaginaires qui peuvent transformer des contrées d'Afrique centrale en territoires où des fils de paysans se retrouvent élevés au rang de « héros »(Campbell, 1987). Leurs hauts-faits sont dorénavant loués tant par les journalistes que par les griots. Dans les fergo les plus connus l'épopée peule de Samba Guéladio ou encore l'équipée de El Hadj Omar - qui font partie du patrimoine oral sénégalais, la recherche d'indépendance morale est exaltée. Le héros conserve sa liberté d'initiative en dépit des suggestions et du poids d'une société holiste. Le fergo se définit à la fois comme l'émigration et la dissidence. L'exil politique doit permettre au retour la reconquête du pouvoir. Quelles que soient les décisions du Satigui Konko, Samba Guéladio passe outre, agissant selon sa volonté propre et c'est parce qu'il tente de se libérer du carcan de la société pulaar qu'il se singularise. De la même manière, El Hadj Omar préfère quitter temporairement le Fuuta Tooro, organisant son armée, débauchant en chemin des volontaires parmi les paysans et fondant ainsi un vaste empire Tukulër plutôt que d'accepter la domination coloniale et de payer l'imposition. Á la tête d'une armée de 30000 hommes et, pour convertir à l'islam les populations animistes, il s'attaque à la région aurifère du Bambouk et au Kaarta avant de conquérir Nioro du Sahel en 1854.

À l'orée de la décolonisation, les jeunes diamantaires en puissance, ressortissants de la vallée du Sénégal, qui s'engagent dans la course et le négoce des pierres brutes, rejouent, à leur niveau, la même partition (Bredeloup, 2004). Au cours de cette épopée, ils s'efforcent de conserver leur libre-arbitre et contestent la soumission aux aînés. Leur aventure se révèle d'abord individuelle ; elle correspond à un désir d'émancipation, à un rejet des contraintes familiales ou statutaires jugées trop rigides; elle est le signe à la fois d'un refus de l'immobilité à laquelle les condamne une société dominée par une gérontocratie toute puissante et d'une soif de découvrir de nouveaux horizons. "Quand j'ai voulu partir en réalité, mes frères étaient opposés. Ils ne voulaient pas me laisser partir parce que d'abord j'étais jeune, j'avais 17 ans... Je pensais faire moi aussi l'aventure dans la vie. L'envie de partir m'empêchait d'être raisonnable et de travailler avec mes frères ${ }^{15}$. Mais leur aventure n'est pas pour autant totalement transgressive puisque à terme, la réussite personnelle ne doit pas empêcher une réintégration dans le milieu d'origine ; elle ne remet pas fondamentalement en question la hiérarchie communautaire.

Cette aventure suppose toutefois une grande dose de témérité, de bravoure et de fierté. À l'instar du héros pulaar, le migrant doit être en mesure de surmonter les épreuves grâce à son courage à la fois physique et moral, grâce à «son aptitude à envisager un danger immédiat sans pour autant dévier de la ligne de conduite qui le fait naître » (Goffman, 1974 : 165). Il ne peut admettre qu'on lui refuse le droit de migrer et de participer à la ruée vers le diamant. Il ne peut supporter d'échouer et de rentrer bredouille au pays. Il force le destin, persévérant dans son entreprise, supportant la souffrance qu'elle provoque pour se préparer un retour glorieux. Il fait preuve de cran, «capable de s'en tenir opiniâtrement à une activité au mépris des revers, des souffrances ou de l'épuisement, non pas avec l'insensibilité de la brute mais par suite d'une détermination intime »(Goffman, 1974 : 165). L'espoir sans limites de changer leur destin par eux-mêmes et par la grâce de Dieu confère à ces diamantaires une force inébranlable qui leur permet d'affronter les dangers et de supporter les obstacles avec sang froid. Expulsion, vol, emprisonnement et assassinat participent des risques du métier. Dans notre enquête menée en 1994 auprès de 376 diamantaires originaires de la vallée du fleuve Sénégal, une dizaine d'entre eux sont décédés entre le début et la fin de nos observations (Bredeloup, 2007). Pour un sur quatre, le dernier retour au village fut le résultat

\footnotetext{
${ }^{15}$ Entretien réalisé à Dakar, le 10 août 1997 avec un trafiquant de diamants.
} 
d'une expulsion. Dans tous les récits de vie recueillis, il en est question, sans exception. Les uns en parlent avec pudeur ; les autres l'évoquent de manière romanesque, dans l'intention de désamorcer le malaise ou encore dans l'espoir que leurs aventures puissent être immortalisées par le chercheur perçu alors comme le nouveau griot des temps modernes. "À l'époque [en 1962], il y avait beaucoup de rebelles dans la région [Zaïre]. Albert Kalodje, le roi de Bacongo ne reconnaissait pas le gouvernement central. Les trafiquants étaient aussi traqués par l'armée régulière... L'année suivante, nous sommes partis à Luluabourg dans une forêt. $C$ 'était une véritable brousse; nous construisions nos chambres avec nos coupe coupes. Nous combattions les militaires. Notre vie était partagée entre la guerre contre les militaires le jour et la nuit, le ramassage des pierres que les rebelles nous autorisaient à prendre ${ }^{16}$. Une fois de plus, cette mise en intrigue conduit à la conversion du migrant en aventurier voire en héros plus ou moins réaliste.

L'aventure renvoie donc une perception guerrière : "Dans beaucoup de pays, nous sommes victimes d'agressions, d'intimidation tant de la part des citoyens que des gouvernements. Alors tous ceux qui n'ont pas de courage retournent, mais ceux qui en ont, ont continué. On nous arrête, on nous emprisonne, on nous torture mais ça ne nous empêche pas d'avancer; c'était une véritable guerre » conclut un trafiquant sénégalais après avoir relaté sa fuite rocambolesque dans la forêt congolaise alors qu'il avait à ses trousses une patrouille de l'armée ${ }^{17}$. Selon le Littré qui se réfère aux chevaliers errants et aux mercenaires à l'époque du Moyen Age, les aventuriers étaient « ceux qui faisaient la guerre en volontaires et sans recevoir de solde ».

\section{Imaginaire du savoir-vivre}

Cette figure pionnière du trafiquant de diamants trouve un écho important aujourd'hui auprès de ceux qui prennent la route vers l'Europe, avec la ferme intention de « sublimer leur vie », c'est-à-dire de triompher de la monotonie journalière qui assaille la majorité des cités africaines, pour devenir acteurs de leur destinée alors que l'Afrique est plongée en pleine crise des référents identitaires. Cette tendance à l'émancipation par la migration, à l'affirmation de son identité propre et son désir d'une plus grande autonomie avait déjà été repérée par I. Barden au seuil des années 1990 alors qu'elle reconstituait les itinéraires de jeunes immigrés ouest-africains se «débrouillant » dans la capitale burkinabè. De son côté, E. Goldschmidt, qui s'est intéressé plus récemment aux migrants congolais arrêtés au Maroc mais en route vers l'Europe, montre aussi que la remontée du continent noir par étapes prend l'allure d'une épreuve initiatique. Ceux qui y parviennent estiment qu'ils ont "mangé le crocodile », c'està-dire qu'ils ont triomphé de l'animal, des obstacles. La fréquentation des frontières de la mort donne un nouveau sens à la vie ; l'épreuve fabriquant du sacré (Le Breton, 1991). Les migrants africains rencontrés en Tunisie ou en Libye explorent concrètement de nouveaux modèles d'être et de faire, prennent des risques pour construire de façon autonome, parfois inédite et innovante leur place dans la société. Autrement dit, revendiquer l'aventure, c'est faire savoir qu'on recherche certaines valeurs en soi et non plus autour de soi, dans la famille, dans la société, institutions qui, dans leur configuration actuelle, ne permet plus véritablement un renouvellement identitaire.

Les jeunes Ivoiriens, décrits par E. De Latour, qui inscrivent leur quotidien, dans les imaginaires de la «civilisation matérielle du succès », revisitent aussi le monde occidental et

\footnotetext{
${ }^{16}$ Entretien, op.cit.

${ }^{17}$ Entretien réalisé à Dakar, le 28 février 1996, avec un trafiquant de diamants.
} 
les valeurs accordées à la technologie et à la mode, réinventent le temps et l'espace d'abord entre eux, à partir d'Abidjan, sur les écrans de télévision et de cinéma ou à l'occasion de fêtes. Á noter que ces imaginaires sont de plus en plus déterritorialisés (Appadurai, 2001), dans le sens où l'Ailleurs caractérise avant tout un espace imaginaire et ne nécessite pas nécessairement l'éloignement géographique (Fouquet, 2007). Toutes ces mythologies retravaillées sur le continent africain, tous ces repères ré-enchantés sont aussi fondateurs du voyage et des traversées à venir.

En porte-à-faux sur le marché de l'emploi, reniant les contraintes du travail et les règles de l'entreprise, les jeunes migrants, qui revendiquent l'aventure, portent aux nues le modèle du self made man. Il n'est pas question de progresser par étapes, d'attendre son heure et d'accumuler du capital : l'emballement est de rigueur, l'impatience, l'enthousiasme et la créativité semblent être les moteurs de ces entrepreneurs d'un nouveau genre. L'argent se doit d'être «flambé » aussi facilement qu'il est gagné. Parmi ces aventuriers, quelques-uns se sont arrêtés à Marseille (Kone, 1997 ; Bertoncello \& Bredeloup, 2004). Originaire principalement de villes de Côte d'Ivoire, du Cameroun, du Congo et de la République Centrafricaine, cette nouvelle génération de migrants aspire à une réussite sociale spectaculaire et instantanée. Loin de passer inaperçus dans les rues piétonnes de la cité phocéenne, les aventuriers arborent Rayban et téléphone portable et interpellent le passant, lui proposant de signer des pétitions diverses, activités plus connues sous le vocable «36 $15 »$.

Assurément, l'aventure demeure aux antipodes de l'ennui et du sérieux. Partir à l'aventure, «partir pour le bout de la terre ${ }^{18}$, c'est mobiliser une philosophie de l'action, c'est aussi privilégier l'intensité de chaque instant qui s'écoule, tout abandonner pour se chercher soi, pour découvrir, à l'occasion, de nouvelles familles métaphoriques (dahira, rap) autrement plus stimulantes que les solidarités traditionnelles en cours de délitement qui ont déjà largement été expérimentées. Atteindre le monde occidental ou rentrer au pays en héros ? Où se situe, en définitive, la sortie de l'aventure ?

\section{L'aventure migratoire : une étape dans la vie?}

\section{L'aventure rime avec jeunesse}

Invariablement, le goût pour l'aventure est présenté aussi bien par les migrants euxmêmes que les chercheurs comme un désir de jeunesse qui devrait cesser de se manifester avec le passage à l'âge mûr. Ces lieux communs ont été renforcés en Europe par les modèles littéraires défendus par le courant romantique. "Parce que la jeunesse serait naturellement ignorante, elle serait prédisposée aux aventures. Et parce que l'esthétique romantique valorise une certaine ignorance, les aventures prennent une valeur nouvelle. - d'autant que le romantisme - , on le sait, s'identifie pour une grande part à l'âge nouveau de l'adolescence », (Venayre, 2002 ; 73). Dans la plupart des romans d'aventure écrits en Occident à destination de la jeunesse, l'aventure est identifiée précisément à une folie de jeunesse et s'achève dès lors que le héros, en se mariant, accède à l'âge adulte. Le jeune homme écervelé se reconfigure définitivement en homme rangé. Pour Simmel, également, "l'aventure ne cadre pas avec le style de vie de la vieillesse ... elle est par excellence la forme de vie qui convient le plus à la jeunesse ». Ce qui relie la jeunesse à l'aventure tiendrait dans l'intensité de la tension avec laquelle elle nous fait ressentir la vie. «Ce qu'on appelle la subjectivité de la

\footnotetext{
${ }^{18}$ En référence au texte de S. Laacher, « Partir pour le bout de la terre ».
} 
jeunesse, c'est tout simplement le fait que les matériaux de la vie, dans leur signification objective, le cèdent en importance au processus qui les entraîne, à la vie même ».

Si pour les sapeurs parisiens, les années passent et le nombre de cycles se répètent, l'aventure demeure également bornée. "Il n'est pas dit qu'un aventurier le reste tout au long de sa vie, nous vivons notre jeunesse »(Gandoulou, op.cit.). L'aventure migratoire est bien envisagée comme une étape dans la vie d'un homme au même titre que son installation. De la même manière, ceux que les villageois du fleuve Sénégal rebaptisent aujourd'hui aventuriers, désignent ces jeunes qui se lancent dans le trafic des pierres précieuses du côté de l'Angola mais qui n'entretiennent pas de liens privilégiés avec leurs compatriotes vieillissants, hier eux-mêmes aventuriers, dorénavant diamantaires, négociants ou retraités. Là encore, pour ces Africains, l'aventure a un temps.

\section{Les sorties de l'aventure}

L'aventure a donc un commencement et une fin. On sortirait de l'aventure comme on y serait entré, avec obligation de réussir selon certains principes édictés dans chaque société et selon l'époque. Au temps des projets aventureux doit succéder la construction de sa carrière. Dans le monde de la Sape notamment, les anciens aventuriers sont considérés comme des «affranchis ». Quelques-uns sont parvenus à mener une vie régulière en banlieue parisienne et à s'intégrer dans la société française par le biais d'une activité professionnelle ou un mariage. Cette émancipation va de pair avec une notoriété symbolique dans le milieu aventurier et la confirmation d'avoir atteint le rang d'initié. Dans les années 1980, pour les aventuriers, l'aboutissement visé était une stabilisation dans Paris après plusieurs «descentes » ou encore une intégration dans la société brazzavilloise. Quand l'échec se produit, l'aventurier est rétrogradé dans sa position première de chômeur ou de petit trafiquant.

Parmi les trafiquants de diamants ouest-africains, plusieurs voies de sortie de l'aventure sont également possibles. Vers le haut pour ceux qui sont en mesure de convertir leur capital de mobilité et de se connecter aux réseaux marchands transnationaux ; vers le bas pour les individus épuisés et socialement désaffiliés, qui ne peuvent ni continuer la route, ni rebrousser chemin et qui, tenus à distance par leurs compatriotes, ont de fortes chances de sombrer dans la folie. Si le départ en migration leur a permis de se forger le caractère, il a offert à quelques-uns seulement la possibilité de préparer un retour héroïque. Selon la tradition pulaar, plus l'exil dure, plus les retrouvailles ont des chances d'être chaleureuses ; cet éloignement préparant la réconciliation entre le héros et son peuple. Une minorité a réussi à s'élever dans la hiérarchie, approvisionnant l'Europe en pierres précieuses ou créant un comptoir sur les rives du fleuve Congo alors que la grande majorité continuait à prendre de gros risques à proximité des zones d'extraction. Pour certains, l'aventure s'est arrêtée en Afrique de l'Ouest où ils végètent encore comme masta $^{19}$ du côté de Séfadu (Sierra Leone) ou de Séguéla (Côte d'Ivoire) pendant que d'autres poursuivaient leur route jusqu'au Congo, dans l'intention d'y consolider leur fortune. Plutôt riches et parfois puissants, ces derniers ont alors investi les quartiers huppés des capitales congolaise, burundaise, zambienne et angolaise, acquérant des villas luxueuses, mariant les filles de l'élite locale. Mais la majorité s'est reconvertie dans le commerce de tissus ou de produits alimentaires toujours en Afrique centrale mais dans des régions moins dangereuses. Enfin, si quelques-uns sont rentrés de migration au bon moment, se donnant les moyens de réinvestir, la plupart des diamantaires sont restés trop longtemps à l'étranger pour comprendre le fonctionnement de leur pays

\footnotetext{
${ }^{19}$ Responsable d'un groupe de diamineurs.
} 
d'origine et tirer pleinement partie de leur dangereuse entreprise. Autrement dit, pour la grande majorité, loin d'être héroïque, la sortie de l'aventure a débouché sur des faillites retentissantes (Bredeloup, 1994). Et puis, quelques-uns, dont on ne parle plus, n'ont pas su résister à la violence de l'aventure et ont sombré dans le désespoir. Mort sociale, mort physique ? C'est bien la mort qui constitue, comme l'écrit V. Jankélévitch, « le précieux épice de l'aventure ». L'aventure n'est pas concevable sans l'éventualité de la mort. Nombre de migrants se sont égarés en cours de route, «sont tombés dans l'aventure » et ont achevé leur parcours dans les déserts, les forêts ou les détroits.

É. De Latour insiste également sur le caractère contraignant et irréversible de l'aventure migratoire. "L'aventure n'est pas ouverte; elle est cernée par l'obligation de réussite selon ses tracés dans l'imaginaire collectif». Autrement dit, le migrant doit être totalement maître de la fin de son aventure. Son retour au pays doit nécessairement être triomphal, permettre à la fois de se réaliser personnellement, "d'éteindre la dette de vie » envers ses parents et d'appuyer les projets des proches dont le succès lui apportera encore davantage de prestige. Le retour de l'aventurier ne peut être que volontaire pour lui permettre d'accroître sa notoriété, sa puissance et de revêtir les habits du héros. Dans ces conditions, une reconduite à la frontière débouchera inévitablement sur une disqualification du migrant, bref, sur son déshonneur. Elle sera interprétée non pas comme une injustice perpétrée par un appareil politique de plus en plus performant dans son contrôle mais plutôt comme un échec personnel. «Mal revenus » ou encore «maudits », ces éclopés de l'aventure peuvent sombrer dans la folie ou l'alcoolisme, attenter à leur vie à moins qu'ils trouvent l'énergie suffisante pour repartir. "L'échec au voyage est pire que l'immobilité sur place : du pauvre au départ, le guerrier devient minable » (De Latour, 2001; 174). Alors que la clandestinité se contourne, le rejet par les pairs aussi bien dans le pays d'origine que dans les espaces de transit peut détruire. L'aventurier est alors condamné à la mobilité que lui refuse précisément les institutions en érigeant des frontières de plus en plus infranchissables.

\section{Conclusion}

La figure de l'aventurier présente à l'évidence une extrême hétérogénéité. Si les migrants en quête d'aventures sont en grande majorité des jeunes citadins, disposés à prendre des risques pour trouver leur voie, les uns sont analphabètes alors que d'autres sont diplômés de l'université ou encore ont accumulé des qualifications professionnelles. La démarche des uns se veut résolument pionnière, individuelle pendant que d'autres se transforment en têtes de pont de futurs réseaux migratoires. C'est ainsi que Boureima, l'un des tous premiers Burkinabè parti explorer les possibilités d'embauche en Italie du nord, au milieu des années 1980, revendique haut et fort le statut d'aventurier alors que d'autres l'auraient rangé dans la catégorie des travailleurs immigrés ordinaires dans la mesure où, depuis quatorze ans, il travaille dans une usine du côté de Milan ${ }^{20}$. Pourtant il a rebaptisé le bar qu'il a réaménagé dans son quartier d'origine "l'Aventura », en souvenir de ses premiers pas balbutiants en direction de la péninsule italienne, quand il travaillait comme chauffeur dans des ambassades africaines, contestait son salaire et assistait les diplomates dans leurs charges protocolaires, quand le plaisir de la découverte se mêlait aux incertitudes du lendemain. Aujourd'hui, régularisé et installé en famille, il accueille les parents en déroute et grâce à son action constante au sein d'une association d'immigrés, il contribue à la transformation notable de son village dans la province du Boulgou. Autrement dit, les aventuriers sont rarement libres

\footnotetext{
${ }^{20}$ Entretien réalisé en mai 2007.
} 
de toute attache et un dessein, aux apparences individuelles, peut très vite se transformer en projet familial au fil du temps et de la conjoncture. Il serait inexact de penser que les migrants, qui partent souvent seuls à l'aventure, développent de bout en bout un itinéraire solitaire et se définissent comme des êtres exclus notamment d'un ordre familial. Ils ne sont pas tous en rupture de ban. Les uns peuvent être rejoints ultérieurement par des membres de la famille ou du village, à l'image de Boureima mais aussi de la plupart des diamantaires rencontrés; d'autres encore s'associent en cours de route à des compagnons d'aventures pour traverser les déserts, franchir les frontières, partager un logement, formant circonstanciellement des « communautés d'itinérance » (Escoffier, 2006) ou procédant à la construction de familles métaphoriques (De Latour, 2001). D'autres enfin peuvent demander soutien à des parents restés au pays ou déjà partis en migration quand le parcours se transforme en voie sans issue. Mais ce qui les fédère tous, c'est une manière d'être en société, à la fois libre et solidaire, une façon d'envisager la vie, une foi en l'avenir, un besoin irrépressible d'accomplissement de soi. S'aventurer c'est s'évader pour chercher la vie, la sublimer, pour créer un nouvel espaces de références, pour se construire durablement au-delà de l'étape voire de l'expérience migratoire.

On peut néanmoins se demander pour quelles raisons cette figure est-elle réifiée aujourd'hui dans le champ migratoire et ressurgit-elle si vivement sur la scène contemporaine. Elle serait en définitive le produit d'une construction initiée à la fois par les migrants euxmêmes, les agents du développement, les politiques et les chercheurs. Les Africains entendent donner du sens à leur quête, aux épreuves qu'ils ont traversées et valoriser le choix qu'ils ont fait de partir au bout du monde. Cette auto-désignation leur donne la force de continuer la route, la puissance en sus d'une légitimité. Cette mise en récit de leur parcours, qui est élaborée invariablement selon un scénario identique (une description qui occulte le départ mais qui laisse une place centrale au voyage, aux embûches, aux exploits et aux rencontres), leur permet de transformer cette expérience qui s'écarte de la norme en rite initiatique, en art de vivre et de satisfaire ainsi leur soif de reconnaissance. De leur côté, en nommant les migrants «aventuriers », les agents de développement et responsables d'ONG leur reconnaissent paradoxalement des capacités d'innovation, une liberté d'action dans un monde de plus en plus sécuritaire. En transformant, sur le mode de l'afro-optimisme, les migrants clandestins soumis au hasard des contrôles en héros créatifs, en mettant en avant les valeurs positives de l'aventure, ces structures associatives procèdent ainsi à leur propre déculpabilisation. D'une autre manière, relayées par les médias, les structures politiques qui qualifient les migrants d'aventuriers leur reconnaissent aussi une marge de liberté. Plutôt que de les victimiser, elles les considèrent comme menaçants, les rendant ainsi responsables de multiples forfaits. L'aventurier est ici présenté comme un individu peu scrupuleux, hors-laloi, à la lisière de l'escroc, qui voyage beaucoup, vit d'expédients et est prêt à tout pour réussir son entreprise. Cette version négative de l'aventure participe de la construction de la menace, du processus de criminalisation du migrant et légitime toute forme de répression à venir. L'aventurier devient alors le vagabond, celui dont « l'accès à la mobilité mondiale » est entravé, le voyageur qui n'a pas le droit de devenir touriste mais dont la simple apparition déclenche le trouble, la peur chez le touriste (Bauman, 1999). Enfin, le chercheur en sciences sociales contribue aussi activement à la mise en scène du migrant, à la réification de l'aventurier, à son héroïsation éventuelle en lui offrant la possibilité de raconter et de réenchanter son histoire, alors qu'il se trouve bien souvent fragilisé. 


\section{Références bibliographiques}

Anderson N., 1923, The Hobo, Chicago, University of Chicago Press.

Andrès B., 2004, Problèmes posés par la figure de l'aventurier, Communication au colloque Figures et mythes littéraires des Amériques, UGAM, 20 mai, 9 p.

Appadurai A., 2001, Après le colonialisme. Les conséquences culturelles de la globalisation, Paris, Payot.

Balandier G., 1977, Ruse et politique, Cause commune, Paris, UGE-10/18.

Banegas R., Warnier J.-P., (coord.), 2001, Figures de la réussite et imaginaires politiques, Politique africaine, $\mathrm{n}^{\circ} 82$, Paris, Karthala.

Bauman Z., 1999, Le coût humain de la mondialisation, Paris, Hachette.

Bardem I., 1993, L'émancipation des jeunes; un facteur négligé des migrations internationales, Cahiers des sciences humaines, 29, 2-3, p. 379-393.

Bazenguissa R., 1992, La SAPE et la politique au Congo, Journal des africanistes 62 (1).

Bazenguissa R., MacGaffey J., 1995,Vivre et briller à Paris. Des jeunes Congolais et Zaïrois en marge de la légalité économique, Politique africaine, ${ }^{\circ}$ 57, p. 124-133.

Becker H., 1963, Outsiders : Studies in the Sociology of Deviance, New York, Free Press

Bertoncello B., Bredeloup S., 2004, Colporteurs africains à Marseille. Un siècle d'aventures, Paris, Autrement, 167 p. [Coll. Français d'ailleurs, peuple d'ici]

Bredeloup S., 1994. L'aventure des diamantaires sénégalais, Politique Africaine, n56, Entrepreneurs, ajustement et démocratie, p. 77-93.

Bredeloup S., 2004, Les pas d'un marin noir africain et ses ajustements : du port au zinc, in Fournier P., Mazzella S., (s-dir.), Marseille entre ville et ports. Les destins sociaux de la rue de la République, Paris, La découverte, coll. Recherches, p.273-287.

Bredeloup S., 2007, La Diams'pora du fleuve Sénégal. Sociologie des migrations africaines, IRD/PUM, Toulouse, $300 \mathrm{p}$.

Bouly de Lesdain S., 1999, Femmes camerounaises en région parisienne. Trajectoires migratoires et réseaux d'approvisionnement, Paris, Harmattan (Connaissance des hommes), 241 p.

Brückner P., Finkielkraut A. 1982, Au coin de la rue, l'aventure, Paris, Seuil.

Campbell J., 1987, Les héros sont éternels, Paris, Seghers (1 ${ }^{\text {ère }}$ édition, 1949 The hero with a Thousand Faces).

Conrad J., 1982, Lord Jim, Gallimard, coll. Folio (1ère édition, 1900).

David P., 1980, Les navétanes, Nouvelles éditions africaines, Dakar-Abidjan, 525 p.

De Boeck F., 2001, Comment dompter diamants et dollars : dépense, partage et identité au Sud-Ouest du Zaïre (1980-1997), Cahiers africains, Afrika Studies, n 45-46, p. 171-208.

De Latour E., 1999, Les ghettomen. Les processus d'identification par l'illégalité à travers les gangs de rue à Abidjan et San Pedro, Cahiers internationaux de Sociologie, 129, p. 68-83.

De Latour É., 2001. Du ghetto au voyage clandestin : la métaphore héroïque, Autrepart, nº19, p.

De Latour É., 2003, Héros de retour, Critique internationale, nº19, avril, p. 171-189.

Ehrenberg A., 1998, L'âge de l'héroïsme, Cahiers internationaux de Sociologie, vol LXXXV, p. 197224.

Escoffier C., 2006, Communautés d'itinérance et savoir-circuler des transmigrant-e-s au Maghreb, Thèse de sociologie, Toulouse II (s-dir. A. Tarrius).

Fouquet T., 2007, Imaginaires migratoires et expériences multiples de l'altérité : une dialectique actuelle du proche et du lointain, Autrepart $\mathrm{n}^{\circ} 21$, p. 83-98.

Goldschmidt É., 2002, «Migrants congolais en route vers 1'Europe », Temps modernes, 620-621, août-nov., p. 208-239.

Gandoulou J.-D., 1989, Au cour de la sape. Mœurs et aventures des Congolais à Paris, Paris, Harmattan.

Jankelevitch V., 1963.- L'aventure, l'ennui, le sérieux, Paris, Aubier (Montaigne), 223 p.

Kivilu S., A la recherche du paradis terrestre : les Bana Luunda entre le diamant et le dollar, Cahiers africains, Afrika Studies, ${ }^{\circ}$ 45-46, p. 127-169.

Kolonga M., 1979, Kinshasa, ce village d'hier, Kinshasa, Sodimca.

Laacher S., 2003, Partir pour le bout de la terre, Critique internationale, ${ }^{\circ} 19$, avril, p. 157-170.

Le Breton D., 1991. Passions du risque, Paris, Métaillé. 
Le Breton D., 1996, L'aventure. La passion des détours, in L'extrême ailleurs, Paris, Autrement, $\mathrm{n}^{\circ} 160$, p. $15-71$.

Mabanckou A., 1999, Bleu-Blanc-Rouge, Paris, Présence africaine, 224 p.

MacGaffey J., Bazenguissa-Ganga R., 2000, Congo-Paris : Transnational Traders on The margins of the Law, Oxford, James Currey Publishers, $190 \mathrm{p}$.

MacOrlan P., 1998, Le petit manuel du parfait aventurier, Mercure de France, (réédition).

Malaquais, 2001, Arts de feyre au Cameroun, Politique africaine, nº8, p. 101-118.

Mathé R., 1972, L'aventure d'Hérodote à Malraux, Paris/Montréal, Bordas, collection « Univers des lettres, $191 \mathrm{p}$.

Monnier L., Jewsiewicki B., de Villers G., (s-dir.), 2001, Chasse au diamant au Congo/Zaïre, Cahiers africains, Afrika Studies, $\mathrm{n}^{\circ} 45-46,240 \mathrm{p}$.

Ouellet R., 1996, L'Aventurier du hasard. Le baron de Lahontan, Québec, Septentrion, 435p.

Ould Ahmed Salem Z., 2001, Tcheb-tchib et compagnie. Lexique de la survie et figures de la réussite en Mauritanie, Politique africaine, $\mathrm{n}^{\circ} 82$, juin, p. 78 -

Pociello C., 1987, Un nouvel esprit d'aventure, Esprit, avril, p. 95-105.

Roche D., 2002, L'étranger insaisissable (XVII-XVIIIe siècles, Mélanges de l'Ecole française de Rome. Italie et Méditerranée, v. 114, n²,p.837-874.

Sartre J.-P., 1972, La Nausée, Paris, Gallimard, coll.Folio (1 ${ }^{\text {ère }}$ édition 1938).

Simmel G., 1912, L'Aventure, in Mélanges de philosophie relativiste, contribution à la culture philosophique, trad. A. Guillain, Paris.

Simmel G., 2002, La philosophie de l'aventure, Ed L'Arche, coll. tête à tête, 128 p.

Spire A., 1999, De l'étranger à l'immigration. La magie sociale d'une catégorie statistique, Annales de la Recherche en Sciences Sociales, 129, septembre, p.50-56.

Tshibanza M., 1986, Le phénomène creuseur et ses paradoxes, Zaïre-Afrique, ${ }^{\circ}{ }^{206}$, juin-août.

Venayre S., 2002, La belle époque de l'aventure (1890-1920), Revue d'histoire du XIXème siècle, 24, varia.

Venayre S., 2002, La gloire de l'aventure. Genèse d'une mystique moderne. 1850-1940, Paris, Aubier. 